\title{
Polarization control of isolated high-harmonic pulses
}

\author{
Pei-Chi Huang ${ }^{1,2 \star}$, Carlos Hernández-García $\odot^{3}$, Jen-Ting Huang, ${ }^{1}$ Po-Yao Huang ${ }^{1}{ }^{1}$, Chih-Hsuan Lu $u^{1,2}$, \\ Laura Rego ${ }^{3}$, Daniel D. Hickstein ${ }^{4}{ }^{4}$, Jennifer L. Ellis ${ }^{4}$, Agnieszka Jaron-Becker ${ }^{4}$, Andreas Becker ${ }^{4}$, \\ Shang-Da Yang', Charles G. Durfee ${ }^{5}$, Luis Plaja ${ }^{3}$, Henry C. Kapteyn ${ }^{4}{ }^{4}$, Margaret M. Murnane ${ }^{4}$, \\ A. H. Kung ${ }^{1,2}$ and Ming-Chang Chen ${ }^{1,6 \star}$
}

\begin{abstract}
High-harmonic generation driven by femtosecond lasers makes it possible to capture the fastest dynamics in molecules and materials. However, thus far, the shortest isolated attosecond pulses have only been produced with linear polarization, which limits the range of physics that can be explored. Here, we demonstrate robust polarization control of isolated extreme-ultraviolet pulses by exploiting non-collinear high-harmonic generation driven by two counter-rotating few-cycle laser beams. The circularly polarized supercontinuum is produced at a central photon energy of $33 \mathrm{eV}$ with a transform limit of 190 as and a predicted linear chirp of $\mathbf{3 3 0}$ as. By adjusting the ellipticity of the two counter-rotating driving pulses simultaneously, we control the polarization state of isolated extreme-ultraviolet pulses-from circular through elliptical to linear polarization-without sacrificing conversion efficiency. Access to the purely circularly polarized supercontinuum, combined with full helicity and ellipticity control, paves the way towards attosecond metrology of circular dichroism.
\end{abstract}

U ltrafast extreme-ultraviolet (EUV) and soft X-ray beams carrying spin angular momentum are used in many different fields of science due to their ability to temporally investigate the structural, electronic and magnetic properties of materials. For example, ultrafast circularly polarized beams are particularly important in the study of chiral molecules, making it possible to discriminate between enantiomers and resolve their dynamics. Bright ultrafast beams with adjustable polarization have recently been produced using both free-electron laser (FEL) facilities $^{1-4}$ and tabletop high-harmonic generation (HHG) set-ups ${ }^{5-9}$. Several schemes to generate circularly polarized beams have been implemented at X-ray FELs, including converting linearly polarized beams to circularly polarized beams using a phase retarder, or by using special undulators to precisely control the motion of electrons, and thus the polarization of the emitted radiation. FEL $\mathrm{X}$-ray pulses are emitted with pulse durations as short as tens of femtoseconds ${ }^{10}$. However, although FEL radiation is well known for its brightness, the high cost of such large-scale facilities results in limited access.

A tabletop alternative to FELs is the extreme nonlinear optical process of HHG, where infrared light from a femtosecond laser can be coherently upconverted to produce fully coherent beams in the EUV and soft X-ray regimes with sub-femtosecond or attosecond pulse durations. HHG is a non-perturbative process that has both microscopic and macroscopic character. Microscopically, an atom is first ionized by the driving field, then the electronic wave packet is accelerated and finally driven back to recollide with the parent ion $^{11,12}$. In this last step, the kinetic energy acquired from the laser field is released as high-frequency harmonics of the fundamental laser. Macroscopically, the phase relationship between harmonics radiated from different atoms in the target becomes crucial for determining both the efficiency and the spatio-temporal properties of the emitted coherent radiation ${ }^{13-17}$.
Although tremendous progress has been made in generating and using linearly polarized high-harmonic beams, until recently it was not possible to directly generate bright circularly polarized HHG pulses. This is because when HHG is driven by a linearly polarized laser, the ionized electronic wave packet accelerates on a linear trajectory and therefore easily recollides with the parent ion. In contrast, with circularly polarized driving lasers (or elliptically polarized lasers with large ellipticity), the probability of electron recollision is strongly suppressed and therefore no harmonics are emitted $^{18,19}$. Alternatively, EUV waveplates could be used to convert linearly polarized harmonics to circular polarization; however, this is extremely inefficient and is restricted to narrow bandwidths ${ }^{20}$.

Recent work has demonstrated the synthesis of bright circularly polarized high-harmonic attosecond pulse trains by modifying the microscopic physics of HHG through the combination of collinear counter-rotating beams with different frequencies and macroscopic phase matching ${ }^{5,6,8,9,21}$. More recently, by using non-collinear counter-rotating circularly polarized driving lasers for HHG (NCPHHGs), circularly polarized trains of attosecond pulses were experimentally generated that emerge as angularly separated beams ${ }^{22}$. Furthermore, numerical simulations suggest that isolated attosecond bursts of circularly polarized light can be generated in NCP$\mathrm{HHG}$, provided that the driving laser pulse duration is reduced to the few-cycle regime ${ }^{23}$.

Here we experimentally generate circularly polarized highharmonic EUV supercontinua, spanning photon energies from 20 to $45 \mathrm{eV}$, and thus supporting 190 as (transform-limited) isolated pulses. As discussed in more detail in the following, although the single isolated high-harmonic burst is predicted to be linearly chirped to $\sim 330 \mathrm{as}$, it represents, to our knowledge, the broadest coherent circularly polarized supercontinuum generated so far. This experiment is realized by the use of few-cycle pulses synthesized from compressed multiple thin-plate supercontinua

IInstitute of Photonics Technologies, National Tsing Hua University, Hsinchu, Taiwan. ${ }^{2}$ Institute of Atomic and Molecular Sciences, Academica Sinica, Taipei, Taiwan. ${ }^{3}$ Grupo de Investigación en Aplicaciones del Láser y Fotónica, Departamento de Física Aplicada, University of Salamanca, Salamanca, Spain. ${ }^{4}$ JILA - Department of Physics, University of Colorado and NIST, Boulder, CO, USA. ${ }^{5}$ Department of Physics, Colorado School of Mines, Golden, CO, USA. 6Department of Physics, National Tsing Hua University, Hsinchu, Taiwan. *e-mail: 19xas9966514@gmail.com; mingchang@mx.nthu.edu.tw 


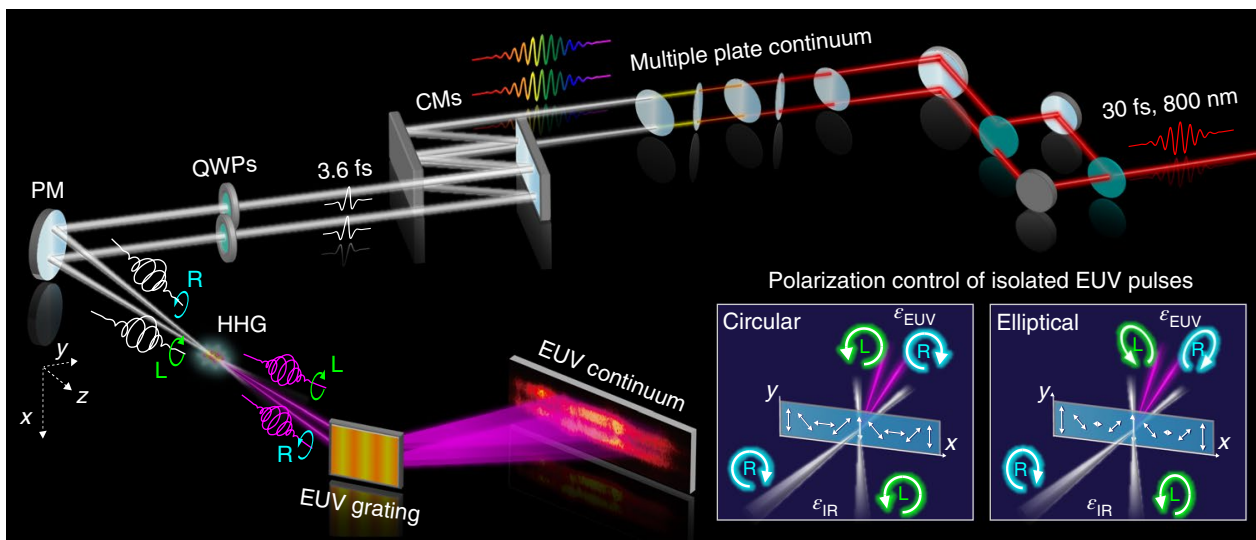

Fig. 1 | Set-up for generating arbitrarily polarized isolated high-harmonic pulses using few-cycle driving lasers. Controlling the ellipticity of the driving lasers results in coherent control of the amplitude and phase of the linearly polarized HHG fields at the focal plane, resulting in polarization control of the HHG beams in the far field. CM, chirped mirror; QWP, quarter-wave plate: PM, off-axis parabolic mirror. Insets: electric field distributions at the focal plane.

(MPContinua) ${ }^{24}$. The unique scalability of the MPContinuum technique enables the realization of almost identical near-single-cycle driving pulses without using split mirrors or a second compressor set-up. To characterize the isolated circularly polarized high-harmonic pulses, the pulse temporal contrast is characterized using an EUV Fourier-transform field autocorrelation, which is a reliable technique to distinguish an isolated EUV pulse from a train of such pulses $^{25}$. Most importantly, we show that this work provides the first polarization control of isolated high-harmonic pulses. We smoothly control the ellipticity of isolated EUV pulses, from linear through elliptical to circular polarization, while retaining the conversion efficiency. Remarkably, the polarization control is based on the nonperturbative nature of $\mathrm{HHG}$, as we unequivocally demonstrate that the polarization state of the isolated EUV pulses depends strongly on the quantum trajectory followed in the HHG process. The polarization state of the isolated high-harmonic pulses is fully analysed with a rigorous and reliable polarimeter composed of two rotatable sets of triple-reflection polarization analysers, which unambiguously determine their ellipticity (the ratio of the minor axis to the major axis of elliptically polarized light), helicity (left-handed or right-handed circular polarization) and degree of polarization (the energy portion of pulses that is polarized). The generation and characterization of arbitrarily polarized high-harmonic pulses represents a major breakthrough in attosecond science, enabling the real-time measurement of energy and angular momentum transfer in magnetic materials and the symmetry-dependent characteristics of chiral molecules.

\section{Polarization control of isolated high-harmonic pulses}

To control the ellipticity of high-harmonic EUV pulses $\varepsilon_{\mathrm{EUV}}$, two identical elliptically polarized few-cycle fundamental laser beams with equal ellipticity $\varepsilon_{\mathrm{IR}}$ (but opposite helicity) were prepared and focused into a gas jet in a non-collinear geometry (Fig. 1). These two electric fields can be written as

$$
\begin{gathered}
E_{\mathrm{IR}, \mathrm{R}}(x, t-D)=\frac{e^{-i(+k z \cos \theta+k x \sin \theta)}}{\sqrt{1+\varepsilon_{\mathrm{IR}}^{2}}}\left[\varepsilon_{\mathrm{IR}} \hat{x}+i \hat{y}\right] \\
E_{\mathrm{IR}, \mathrm{L}}(x, t)=\frac{e^{-i(+k z \cos \theta-k x \sin \theta)}}{\sqrt{1+\varepsilon_{\mathrm{IR}}^{2}}}\left[\varepsilon_{\mathrm{IR}} \hat{x}-i \hat{y}\right] \\
e^{-2 \ln 2\left(\frac{t}{\tau}\right)^{2}+i \omega t+i \Phi_{\mathrm{CEP}}}
\end{gathered}
$$

where $E_{\mathrm{IR}, \mathrm{R}}\left(E_{\mathrm{IR}, \mathrm{L}}\right)$ represents right-handed (left-handed) elliptically polarized infrared (IR) light, $k=2 \pi / \lambda_{\mathrm{IR}}$ is the fundamental wavevector, $\lambda_{\mathrm{IR}}$ is the wavelength of the driving laser, $\theta$ is the halfcrossing angle between the two fundamental laser beams, $x$ and $z$ are the transverse and longitudinal distances away from the centre of the focal plane, respectively, $\tau$ is the full-width at half-maximum (FWHM) pulse duration, $D$ is the delay between these two Gaussian-shaped electric fields, $\omega$ is the angular frequency of the driving laser, and $\Phi_{\mathrm{CEP}}$ is the carrier-envelope phase of the fundamental pulses.

At the focal plane $(z=0)$ and for perfect temporal overlap $(D=0)$, the electric field $E_{\text {focus }}$ becomes

$$
\begin{aligned}
& E_{\text {focus }}(x, t)=E_{\mathrm{IR}, \mathrm{R}}(x, t)+E_{\mathrm{IR}, \mathrm{L}}(x, t) \\
& =\frac{2}{\sqrt{\left(1+\varepsilon_{\mathrm{IR}}^{2}\right)}}\left[\varepsilon_{\mathrm{IR}} \cos (k x \sin \theta) \hat{x}+\sin (k x \sin \theta) \hat{y}\right] \\
& e^{-2 \ln 2(t / \tau)^{2}+i \omega t+i \Phi_{\mathrm{CEP}}}
\end{aligned}
$$

Therefore, $E$-field vectors are created on the focal plane rotating across the transverse direction $\widehat{x}$ with a period of $2 \pi /(k \sin \theta)$. For any specific position $x$, the local $E$-field oscillates linearly, which is ideal for efficient single-atom HHG. In the far-field, the high-harmonic field distribution and vector varying with time can be numerically calculated with the 'thin slab model' (TSM) ${ }^{26,27}$, a semiclassical method based on Fraunhofer diffraction (see Supplementary Section 2 for details). The diffraction integral of the linearly polarized oscillating high-harmonic field distributed along $\widehat{x}$ (Fig. 1) results in two elliptically polarized EUV beams. Because both the amplitude and phase of HHG on the focal plane are coherently scaled and spatially imprinted by the driving IR field, $\varepsilon_{\mathrm{EUV}}$ can be well controlled by $\varepsilon_{\mathrm{IR}}$, forming the ellipticity scaling as $\varepsilon_{\mathrm{EUV}} \propto \varepsilon_{\mathrm{IR}}^{4.5}-\varepsilon_{\mathrm{IR}}^{4.8}$, as discussed in more detail in the section 'Complete polarization control of isolated EUV pulses'.

\section{Isolated, circularly polarized EUV pulses}

The output of a Ti:sapphire amplifier ( $30 \mathrm{fs}, 1 \mathrm{kHz}, 480 \mu \mathrm{J})$ was split into two beams using a slightly misaligned Mach-Zehnder interferometer (Fig. 1). Both linearly polarized laser beams were focused into an array of individual thin quartz plates of $50 \mu \mathrm{m}$ thickness to coherently broaden the pulse bandwidth (Supplementary Section 1 and ref. ${ }^{24}$ ). The resulting MPContinua were simultaneously compressed using the same chirped mirrors to $\sim 3.6 \mathrm{fs}$ ( 1.3 cycles), as confirmed by polarization gating cross-correlation frequency-resolved optical gating ${ }^{28}$. The NCP-HHG was implemented by inserting quarter 

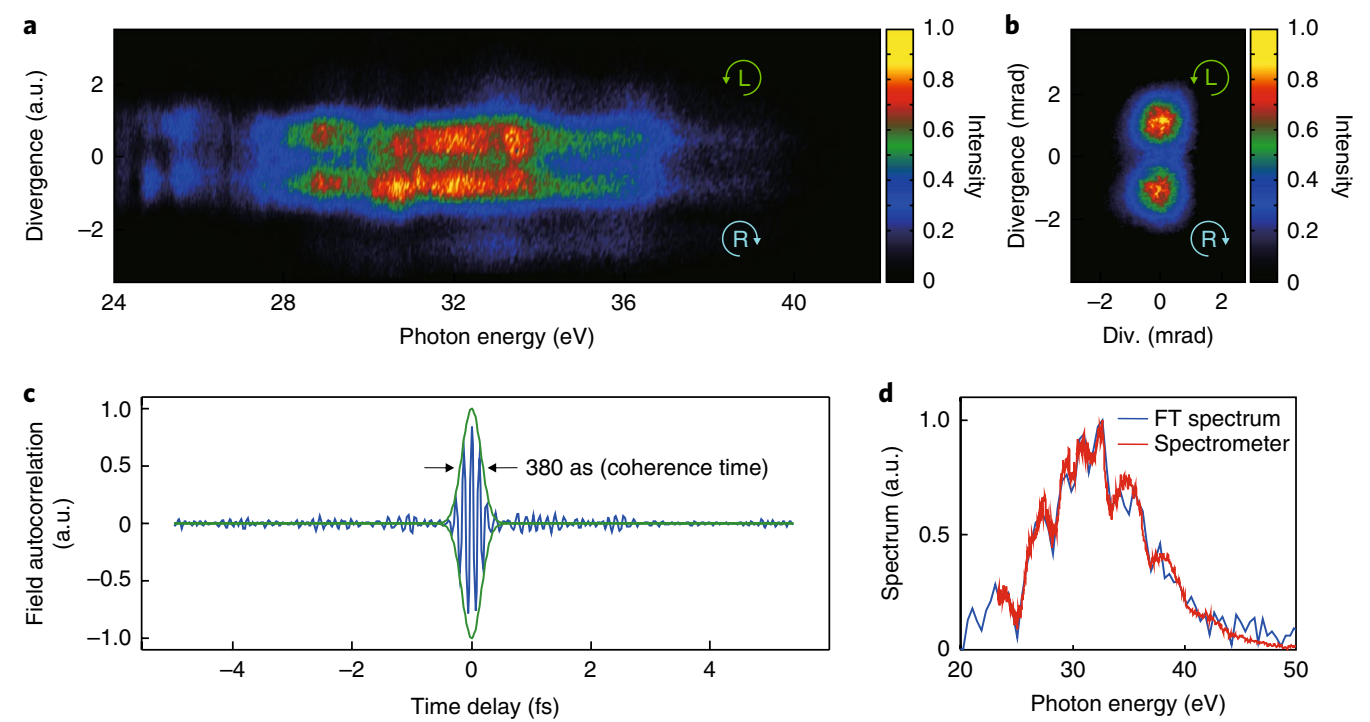

Fig. 2 | Experimental demonstration and characterization of circularly polarized EUV HHG supercontinua. a, Upper and lower spectra are left- and rightcircularly polarized HHG supercontinua with $\varepsilon_{\mathrm{EUV}, \mathrm{L}}=0.93$ and $\varepsilon_{\mathrm{EUV}, \mathrm{R}}=0.9$. b. Beam profiles. Their normalized intensity scales are represented in the colour bar. c, Experimental field autocorrelation trace of circularly polarized HHG pulses (Supplementary Video 1). d, Circularly polarized EUV spectrum measured by a grating-dispersed spectrometer (red), compared with the Fourier transform of the field autocorrelation trace (blue).

waveplates into the two laser beams to produce counter-rotating polarization states. The two laser beams were then focused in a crossed-beam geometry onto a continuous-flow Ar gas jet placed in a vacuum chamber. The half-crossing angle was $\sim 17 \mathrm{mrad}$, and the pulse energies were $\sim 70 \mu \mathrm{J}$. Two $200 \mathrm{~nm} \mathrm{Al}$ filters were used to reject the residual laser light, and the HHG output was spectrally dispersed using an EUV spectrometer, calibrated to better than $0.3 \mathrm{eV}$ resolution. When both driving pulses overlapped in time and space, a continuous high-harmonic spectrum was observed (Fig. 2), because both driving pulses are near single cycles. To optimize the EUV continuum, the two counter-rotating driving pulses were delayed with respect to each other, to narrow the linear-polarization temporalgating window and thus obtain an isolated high-harmonic pulse $\mathrm{e}^{23,29}$ (Supplementary Section 3). Figure 2a presents the spatially resolved high-harmonic supercontinua for a relative time delay of $1.8 \mathrm{fs}$. The two HHG spectra with right and left circular polarization obtained with the NCP-HHG technique are clearly distinguishable. The generated photon flux of each beam is $\sim 3 \times 10^{7}$ counts per second over the whole EUV spectrum. Note that low-order harmonics below $24 \mathrm{eV}$ are mainly reabsorbed in the Ar generating medium.

To measure the temporal coherence and to distinguish the generation of an isolated EUV pulse from a train of pulses, we implemented a Fourier-transform field autocorrelation, as has previously been done for linearly polarized pulses ${ }^{25}$. One of the circularly polarized EUV beams was spatially selected and sent to a split mirror, separating the beam into two halves. A piezo actuator controlled the relative time delay between the two halves of the beam with sub-nanometre resolution. The resulting interference pattern was measured by an EUV charge-coupled device (CCD) as a function of time delay, providing the autocorrelation trace of the HHG beam (Supplementary Video 1). The HHG autocorrelation trace in Fig. 2c shows that the main EUV pulse has a high-amplitude contrast compared to the adjacent pulses and a coherence length of 380 as. Although the carrier-envelope phase $\left(\Phi_{\mathrm{CEP}}\right)$ of IR pulses was not stabilized in our experiment, our simulation results demonstrate that isolated high-harmonic pulses are still generated by controlling the linear-polarization temporal-gating window (Supplementary Section 3). Figure $2 \mathrm{~d}$ shows a comparison between the spectrum extracted from a Fourier transform of the field autocorrelation trace and that experimentally measured by the grating-based spectrometer. The consistency between the two spectra indicates that all circularly polarized EUV light has been successfully delivered and spatially overlapped at one focal spot, which is an essential criterion for circularly polarized attosecond pulse generation. The observed bandwidth supports 190 as transform-limited pulses, while our theoretical simulations predict that isolated chirped pulses of 330 as are produced (Fig. 4 and Supplementary Fig. 4). It is worth noting that using a field autocorrelator, we can clearly show that the supercontinuum corresponds to an isolated pulse, while the good spectrum and beam observed indicate high temporal and spatial coherence of the circularly polarized pulses.

\section{Complete polarization control of isolated EUV pulses}

Furthermore, by adjusting the ellipticity $\varepsilon_{\mathrm{IR}}$ of the two counter-rotating driving pulses, we demonstrate full control of ellipticity of isolated high-harmonic EUV pulses, while keeping the high-harmonic beam quality and conversion efficiency (Fig. 3, Supplementary Fig. 8 and Supplementary Table 1). To maintain linearly polarized $E$-fields at the focal plane, as shown in equation (2) and Fig. 1, we prepared two counter-rotating driving pulses with identical elliptical polarization $\varepsilon_{\mathrm{IR}, \mathrm{R}}=\varepsilon_{\mathrm{IR}, \mathrm{L}}=\varepsilon_{\mathrm{IR}}$. The resulted polarization state of the right-handed and left-handed EUV pulses, $\varepsilon_{\mathrm{EUV}, \mathrm{R}}$ and $\varepsilon_{\mathrm{EUV}, \mathrm{L}}$, were measured separately by one reflection polarimeter consisting of two independent rotatable sets of triple-reflection polarizers, with the first set acting as the phase shifter and the second acting as a linear-polarization analyzer as shown in Fig. $3 \mathrm{a}^{30}$. Such a polarimeter unambiguously determines the ellipticity and helicity of the EUV pulses, as well as discriminating between unpolarized and polarized light by retrieving the Stokes parameters (Supplementary Section 7). While those two polarizer sets are set in the same rotating angle $(\alpha=\beta)$, the polarimeter becomes a great EUV polarization analyser with an extinction ratio of $\sim 80: 1$. Figure $3 c$ shows the angularly resolved intensity of the high-harmonic pulses recorded under different orientations of the analyser axis, together with a table showing the ellipticity of the high-harmonic pulses retrieved by the polarimeter. It is worth mentioning that we experimentally observed almost identical ellipticity and tilt over the entire HHG spectra, clearly indicating that full polarization control of a very broad bandwidth of several tens of electronvolts is straightforward with the NCP-HHG geometry (Supplementary Section 8). 

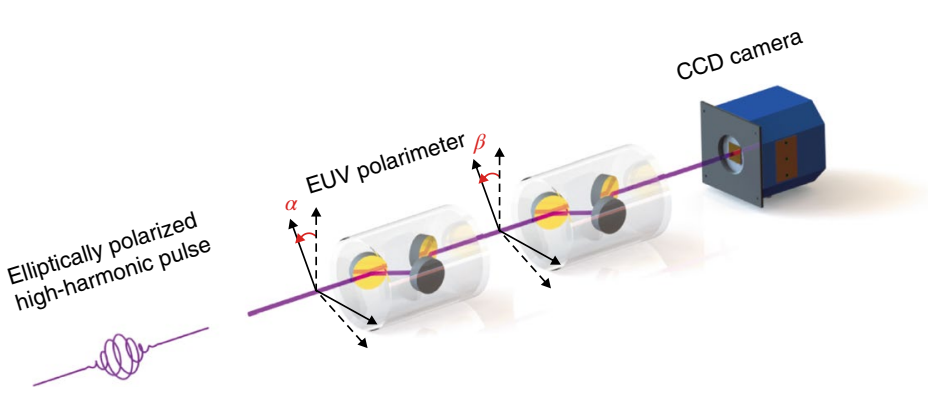

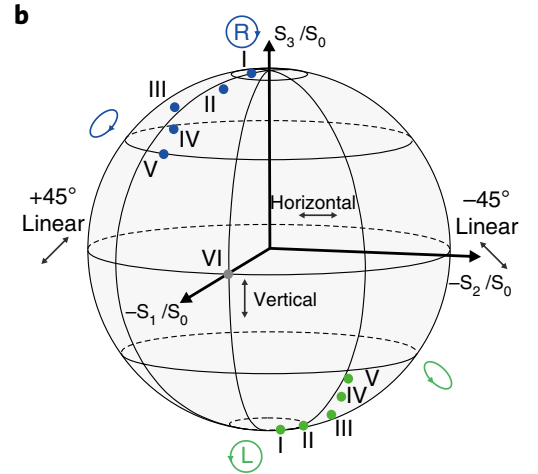

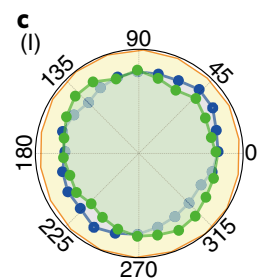
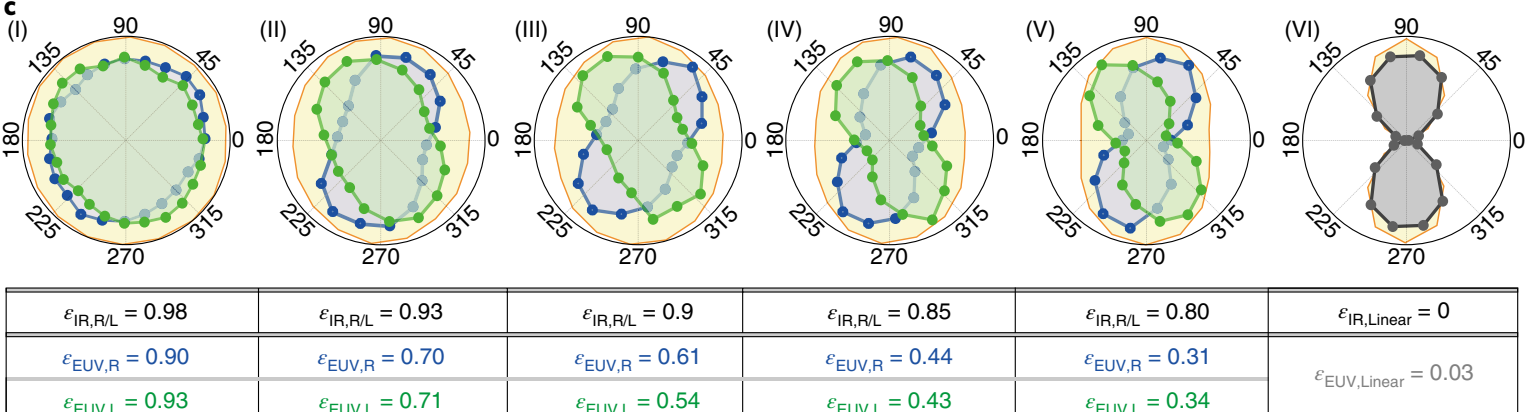

$\varepsilon_{\mathrm{IR}, \mathrm{R} / \mathrm{L}}=0.93$
$\varepsilon_{\mathrm{EUV}, \mathrm{R}}=0.70$
$\varepsilon_{\mathrm{EUV}, \mathrm{L}}=0.71$

\begin{tabular}{c|c|}
\hline & $\varepsilon_{\mathrm{IR}, \mathrm{R} / \mathrm{L}}=0.9$ \\
\hline & $\varepsilon_{\mathrm{EUV}, \mathrm{R}}=0.61$ \\
$\varepsilon_{\mathrm{EUV}, \mathrm{L}}=0.54$
\end{tabular}

\begin{tabular}{|c|}
$\varepsilon_{\mathrm{IR}, \mathrm{R}}$ \\
$\varepsilon_{\mathrm{EUV}}$ \\
$\varepsilon_{\mathrm{EU}}$
\end{tabular}

\begin{abstract}
$=0.85$
\end{abstract}

\begin{abstract}
20
\end{abstract}

\begin{tabular}{|c|c|}
\hline$\varepsilon_{\mathrm{IR}, \text { Linear }}=0$ \\
\hline$\varepsilon_{\text {EUV Linear }}=0.03$ \\
\hline
\end{tabular}

Fig. 3 | Full polarization control of isolated high-harmonic pulses. a, Schematic of the EUV polarimeter used to characterize the polarization state of isolated high-harmonic pulses, where $\alpha$ and $\beta$ are the rotating angles of the two mirror sets, respectively. $\mathbf{b}$, Several polarization states, Stokes parameters $\left(\mathrm{S}_{1} / \mathrm{S}_{0}, \mathrm{~S}_{2} / \mathrm{S}_{0}, \mathrm{~S}_{3} / \mathrm{S}_{0}\right)$, have been generated, characterized and marked on the Poincaré surface. $\mathbf{c}$, The polarization states of the input IR (orange lines) and output EUV (blue and green dots) are measured by one IR and one EUV polarization analyser, respectively, and are depicted in the polar plots. The table gives the ellipticity relation between $\varepsilon_{\mathrm{IR}}$ and $\varepsilon_{\mathrm{EUV}}$. For comparison, linear high-harmonic pulses driven by a linearly polarized fundamental were also measured (grey dots in inset VI).

To verify the working principle behind this method, we performed numerical simulations of the NCP-HHG technique using two theoretical methods: (1) calculations of HHG including propagation, using a combination of the fully quantum strong-field approximation (SFA) and the electromagnetic field propagator ${ }^{31}$; and (2) a semiclassical HHG approximation, the TSM, based on Fraunhofer diffraction ${ }^{26,27}$. Figure 4 a presents $\varepsilon_{\mathrm{EUV}, \mathrm{R} / \mathrm{L}}$ versus $\varepsilon_{\mathrm{IR}}$ for experimental (dots), quantum SFA theory (stars) and TSM results with (pink crosses), and without (yellow crosses) the dipole harmonic phase. We see that the ellipticity scaling obtained by SFA with propagation $\left(\varepsilon_{\mathrm{EUV}} \propto \varepsilon_{\mathrm{IR}}^{4.5}\right)$ or the TSM $\left(\varepsilon_{\mathrm{EUV}} \propto \varepsilon_{\mathrm{IR}}^{4.6}\right)$ agrees well with that observed in the experiments $\left(\varepsilon_{\mathrm{EUV}} \propto \varepsilon_{\mathrm{IR}}^{4.8}\right)$. The excellent agreement allows us to obtain a very straightforward ellipticity control that contrasts with all other approaches for polarization control of high-harmonic pulses so far, which are restricted to narrow bandwidths, low yields or cannot support isolated attosecond pul ses $^{5-9,20,32-35}$. Here, the quantum SFA simulations including propagation were performed using the $3.6 \mathrm{fs}$ driving field as characterized in the experiment, applying a time delay between the non-collinear pulses of $D=+1.8 \mathrm{fs}$ ( +0.7 cycles of IR). The $\varepsilon_{\mathrm{EUV}}$ is calculated for the far-field harmonic signal detected at $\pm 1.0 \mathrm{mrad}$, integrating over a 1 mrad spatial window, mimicking the experiment (for more details of the simulation parameters see Supplementary Section 2).

The experimental results also show that the orientations of the two counter-rotating high-harmonic beams are mirror-symmetrically tilted with respect to the major axis of the fundamental beams (Fig. 3c). Figure 4a presents the scaling of the tilt angle versus $\varepsilon_{\mathrm{IR}}$ for experiments (dots) and quantum SFA theory (stars). To gain insight into the scalings of the ellipticity and tilt angle, we performed semiclassical simulations using the TSM. One of the advantages of the TSM is that it allows us to identify the role of the intensity-dependent dipole harmonic phase, which is the phase acquired by the electronic wave packet in the microscopic HHG process. In Fig. 4a, the TSM results are plotted including (pink crosses) and deliberately neglecting (yellow crosses) the dipole harmonic phase. The present results correspond to a photon energy of $33 \mathrm{eV}$, which is the central energy of the harmonic pulses, integrated over a $1 \mathrm{mrad}$ window in the far-field, centred at $\pm 1.0 \mathrm{mrad}$ (for more details about the TSM calculations see Supplementary Section 2). Our TSM simulations unequivocally show (1) the relevance of the dipole phase in the ellipticity scaling, and (2) that the tilt is induced mainly by the intensity-dependent dipole phase of HHG. Thus, both the ellipticity and tilt angle of the harmonic pulses depend strongly on the quantum trajectory followed in the HHG process. According to equation (2), when the driving fields are elliptically polarized $\left(\varepsilon_{\mathrm{IR}}<1\right)$ at the focal plane, not only does the direction of the linearly polarized E-field rotate, but there is also a field-amplitude modulation with a period of $2 \pi /(k \sin \theta)$ along the transverse $\hat{x}$ direction. Because the dipole harmonic phase depends on the field intensity, such amplitude modulation induces an additional dipole phase modulation along the $\hat{x}$ direction. In short, the field-amplitude modulation on the focal plane actually induces an additional phase shift between the far-field $\hat{x}$ and $\hat{y}$ harmonic-field components, modifying the EUV ellipticity and rotating the major axis of the high-harmonic pulses.

Finally, Figure $4 \mathrm{~b}-\mathrm{d}$ presents numerical simulations of the time evolution of the high-harmonic pulses obtained for $\varepsilon_{\mathrm{IR}}=1.0$ (Fig. $4 \mathrm{~b}$ ), $\varepsilon_{\mathrm{IR}}=0.9$ (Fig. 4c) and $\varepsilon_{\mathrm{IR}}=0.8$ (Fig. $4 \mathrm{~d}$ ), for right-handed (blue) and left-handed (green) helicities, with a time delay of $D=+0.7$ cycles of IR. These results were obtained with our quantum simulations (SFA and propagation) using the $3.6 \mathrm{fs}$ driving field as characterized in the experiment, and a time-gating delay of $1.8 \mathrm{fs}$. As can be observed, isolated attosecond pulses with controlled ellipticity are obtained. Note 

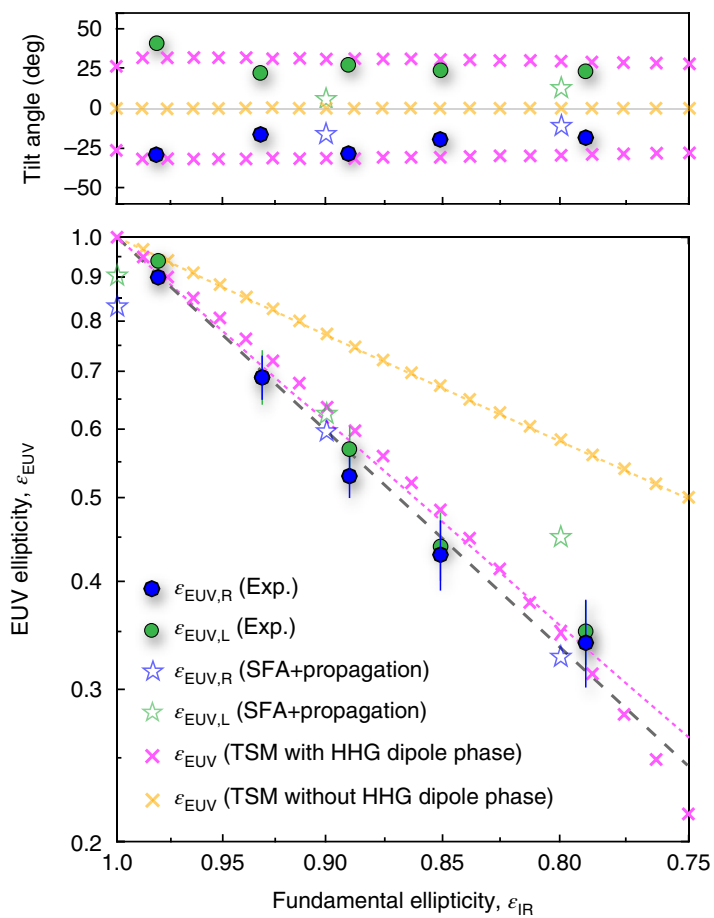

b

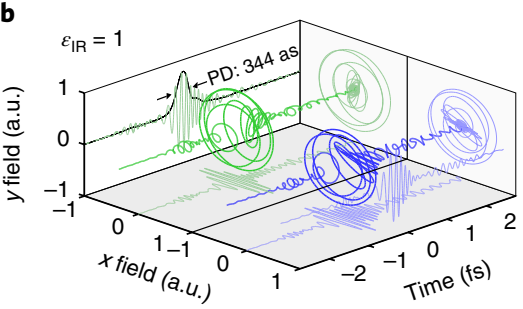

c

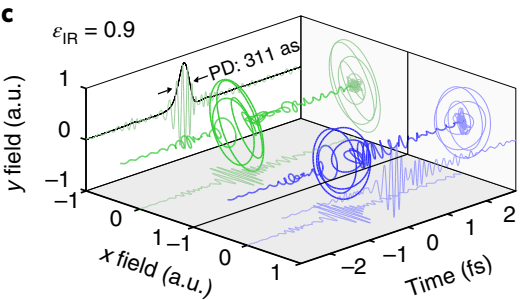

d

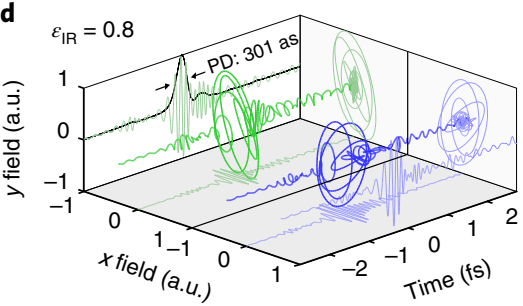

Fig. 4 | Ellipticity and tilt angle scaling of isolated high-harmonic pulses. a, Top: relative tilt angle of the major axis between the EUV pulses and the driving laser versus $\varepsilon_{\mathrm{IR}}$. Bottom: log-log plot of the observed relation between $\varepsilon_{\mathrm{EUV}}$ and $\varepsilon_{\mathrm{IR}}$ (data were obtained from the result in Fig. $3 \mathrm{c}$ and Supplementary Table 1; the error bars represent the standard deviation (thin vertical lines), extracted from 5 individual polarization analyses). The ellipticity scaling can be approximated as $\varepsilon_{\mathrm{EUV}} \propto \varepsilon_{\mathrm{IR}}^{4.8}$ (black dashed line), in excellent agreement with the scaling of the TSM, including the HHG dipole phase (pink short dashed line), $\varepsilon_{\mathrm{EUV}} \propto \varepsilon_{\mathrm{IR}}^{4.6} \cdot \mathbf{b}-\mathbf{d}$, Time evolution of the high-harmonic attosecond pulses obtained using $\varepsilon_{\mathrm{IR}}=1.0$ (b), $\varepsilon_{\mathrm{IR}}=0.9$ (c) and $\varepsilon_{\mathrm{IR}}=0.8$ (d) for the right-handed (blue) and left-handed (green) helicities detected at \pm 1 mrad, respectively. Black lines show the temporal intensity profiles and pulse durations (PDs) of 344,311 and 301 as, respectively.

that each panel is normalized to the same quantity, so the upconversion efficiencies are similar for each $\varepsilon_{\mathrm{IR}}$, agreeing well with our experimental observations (Supplementary Table 1). The simulations also indicate that, for each $\varepsilon_{\mathrm{IR}}$, the pulse durations of resultant circularly or elliptically polarized attosecond pulses are very similar and linearly chirped to approximately 330 as because of the attochirp; however, these could be compressed to near their transform limit of $\sim 190$ as using chirped multilayer mirrors or metal-foil filters ${ }^{36}$.

In summary, we demonstrate a robust polarization control scheme of isolated high-harmonic EUV pulses by exploiting non-collinear HHG driven by two counter-rotating few-cycle laser beams. The circularly polarized supercontinuum is produced in the photon energy range from $20 \mathrm{eV}$ to $45 \mathrm{eV}$, with a high $>0.90$ degree of ellipticity. The isolation and temporal coherence of high-harmonic bursts was measured by EUV interferometry, and the polarization state of the pulses was characterized with an EUV polarimeter. Our simulation results provide clear insight into the three-dimensional electric field vector on attosecond timescales, revealing a pulse duration of $\sim 330$ as. Furthermore, by adjusting the ellipticity of the two counterrotating driving pulses simultaneously, we achieve full control of the polarization state of high-harmonic pulses from circular through elliptical to linear polarization, while retaining the conversion efficiency. We unequivocally identify that the non-perturbative dipole phase of HHG is essential in the ellipticity and tilt angle scaling of HHG pulses, obtaining an ellipticity scaling rule of $\varepsilon_{\mathrm{EUV}} \propto \varepsilon_{\mathrm{IR}}^{4.5}-\varepsilon_{\mathrm{IR}}^{4.8}$. This new technique presents an accessible and reliable route for the generation of isolated arbitrarily polarized attosecond EUV pulses. Because of the importance of this new capability for ultrafast dynamics in angular momentum transfer in atoms, chiral molecules, and $2 \mathrm{D}$ and magnetic materials ${ }^{37-49}$, where very fast dynamics are known to occur and are not currently understood, our work represents an experimental breakthrough with several new aspects and impacts. This work can therefore motivate future work on methods to fully characterize the three-dimensional electric-field vector on attosecond timescales, which is currently a great challenge.

Data availability. The data that support the plots within this paper and other findings of this study are available from the corresponding author upon reasonable request.

Received: 9 November 2017; Accepted: 8 March 2018; Published online: 23 April 2018

\section{References}

1. Spezzani, C. et al. Coherent light with tunable polarization from single-pass free-electron lasers. Phys. Rev. Lett. 107, 084801 (2011).

2. Suzuki, M., Inubushi, Y., Yabashi, M. \& Ishikawa, T. Polarization control of an $\mathrm{X}$-ray free-electron laser with a diamond phase retarder. J. Synchrot. Radiat. 21, 466-472 (2014).

3. Lutman, A. A. et al. Polarization control in an X-ray free-electron laser. Nat. Photon. 10, 468-472 (2016).

4. Allaria, E. et al. Control of the polarization of a vacuum-ultraviolet, high-gain, free-electron laser. Phys. Rev. X 4, 041040 (2014).

5. Eichmann, H., Egbert, A., Nolte, S., Momma, C. \& Wellegehausen, B. Polarization-dependent high-order two-color mixing. Phys. Rev. A 51, R3414-R3417 (1995).

6. Fleischer, A., Kfir, O., Diskin, T., Sidorenko, P. \& Cohen, O. Spin angular momentum and tunable polarization in high-harmonic generation. Nat. Photon. 8, 543-549 (2014). 
7. Ferré, A. et al. A table-top ultrashort light source in the extreme ultraviolet for circular dichroism experiments. Nat. Photon. 9, 93-98 (2015).

8. Kfir, O. et al. Generation of bright phase-matched circularly-polarized extreme ultraviolet high harmonics. Nat. Photon. 9, 99-105 (2015).

9. Fan, T. et al. Bright circularly polarized soft X-ray high harmonics for X-ray magnetic circular dichroism. Proc. Natl Acad. Sci. USA 112 14206-14211 (2015)

10. Ribic, P. R. \& Margaritondo, G. Status and prospects of X-ray free-electron lasers (X-FELs): a simple presentation. J. Phys. D 45, 213001 (2012).

11. Schafer, K. J., Yang, B., DiMauro, L. F. \& Kulander, K. C. Above threshold ionization beyond the high harmonic cutoff. Phys. Rev. Lett. 70, 1599-1602 (1993)

12. Corkum, P. B. Plasma perspective on strong-field multiphoton ionization. Phys. Rev. Lett. 71, 1994-1997 (1993).

13. Balcou, P., Salieres, P., L'Huillier, A. \& Lewenstein, M. Generalized phasematching conditions for high harmonics: the role of field-gradient forces. Phys. Rev. A 55, 3204-3210 (1997)

14. Rundquist, A. et al. Phase-matched generation of coherent soft X-rays. Science 280, 1412-1415 (1998)

15. Gaarde, M. B., Tate, J. L. \& Schafer, K. J. Macroscopic aspects of attosecond pulse generation. J. Phys. B 41, 132001 (2008).

16. Arpin, P., Murnane, M. M. \& Kapteyn, H. C. Quasi-phase-matching of momentum and energy in nonlinear optical processes. Nat. Photon. 4, 571-575 (2010).

17. Sun, H.-W. et al. Extended phase matching of high harmonic generation by plasma-induced defocusing. Optica 4, 976-981 (2017).

18. Dietrich, P., Burnett, N. H., Ivanov, M. \& Corkum, P. B. High-harmonic generation and correlated two-electron multiphoton ionization with elliptically polarized light. Phys. Rev. A 50, R3585-R3588 (1994).

19. Weihe, F. A. et al. Polarization of high-intensity high-harmonic generation. Phys. Rev. A 51, R3433-R3436 (1995).

20. Vodungbo, B. et al. Polarization control of high order harmonics in the EUV photon energy range. Opt. Express 19, 4346-4356 (2011).

21. Medišauskas, L., Wragg, J., van der Hart, H. \& Ivanov, M. Y. Generating isolated elliptically polarized attosecond pulses using bichromatic counterrotating circularly polarized laser fields. Phys. Rev. Lett. 115, 153001 (2015).

22. Hickstein, D. D. et al. Non-collinear generation of angularly isolated circularly polarized high harmonics. Nat. Photon. 9, 743-750 (2015).

23. Hernandez-Garcia, C. et al. Schemes for generation of isolated attosecond pulses of pure circular polarization. Phys. Rev. A 93, 043855 (2016).

24. Lu, C.-H. et al. Generation of intense supercontinuum in condensed media. Optica 1, 400-406 (2014).

25. Chen, M.-C. et al. Generation of bright isolated attosecond soft X-ray pulses driven by multicycle midinfrared lasers. Proc. Natl Acad. Sci. USA 111, E2361-E2367 (2014)

26. Hernández-García, C., San Román, J., Plaja, L. \& Picón, A. Quantum-path signatures in attosecond helical beams driven by optical vortices. New. J. Phys. 17, 093029 (2015).

27. Rego, L., San Román, J., Picón, A., Plaja, L. \& Hernández-García, C. Nonperturbative twist in the generation of extreme-ultraviolet vortex beams. Phys. Rev. Lett. 117, 163202 (2016)

28. Trebino, R. et al. Measuring ultrashort laser pulses in the time-frequency domain using frequency-resolved optical gating. Rev. Sci. Instrum. 68, 3277-3295 (1997)

29. Heyl, C. M. et al. Noncollinear optical gating. New. J. Phys. 16, 052001 (2014)

30. Koide, T. et al. Elliptical-polarization analyses of synchrotron radiation in the 5-80-eV region with a reflection polarimeter. Nucl. Instrum. Methods Phys. Rec. Sect. A 308, 635-644 (1991).

31. Hernández-García, C. et al. High-order harmonic propagation in gases within the discrete dipole approximation. Phys. Rev. A 82, 033432 (2010)

32. Xie, $\mathrm{X}$. et al. Internal momentum state mapping using high harmonic radiation. Phys. Rev. Lett. 101, 033901 (2008)

33. Zhou, X. et al. Elliptically polarized high-order harmonic emission from molecules in linearly polarized laser fields. Phys. Rev. Lett. 102, 073902 (2009).

34. Fleischer, A., Sidorenko, P. \& Cohen, O. Generation of high-order harmonics with controllable elliptical polarization. Opt. Lett. 38, 223-225 (2013).

35. Dorney, K. M. et al. Helicity-selective enhancement and polarization control of attosecond high harmonic waveforms driven by bichromatic circularly polarized laser fields. Phys. Rev. Lett. 119, 063201 (2017).

36. Chini, M., Zhao, K. \& Chang, Z. The generation, characterization and applications of broadband isolated attosecond pulses. Nat. Photon. 8, 178-186 (2014).

37. Stöhr, J. et al. Element-specific magnetic microscopy with circularly polarized X-rays. Science 259, 658-661 (1993).

38. Boeglin, C. et al. Distinguishing the ultrafast dynamics of spin and orbital moments in solids. Nature 465, 458-461 (2010)

39. Meyer-Ilse, J., Akimov, D. \& Dietzek, B. Recent advances in ultrafast time-resolved chirality measurements: perspective and outlook. Laser Photon. Rev. 7, 495-505 (2013).
40. Graves, C. E. Nanoscale spin reversal by non-local angular momentum transfer following ultrafast laser excitation in ferrimagnetic GdFeCo. Nat. Mater. 12, 293-298 (2013).

41. Chefdeville, S. et al. Direct determination of absolute molecular stereochemistry in gas phase by Coulomb explosion imaging. Science 341, 1094-1096 (2013).

42. Bigot, J.-Y., Vomir, M. \& Beaurepaire, E. Coherent ultrafast magnetism induced by femtosecond laser pulses. Nat. Phys. 5, 515-520 (2009).

43. Mangot, L. et al. Broadband transient dichroism spectroscopy in chiral molecules. Opt. Lett. 35, 381-383 (2010).

44. Janssen, M. H. \& Powis, I. Detecting chirality in molecules by imaging photoelectron circular dichroism. Phys. Chem. Chem. Phys. 16, 856-871 (2014).

45. Cireasa, R. et al. Probing molecular chirality on a sub-femtosecond timescale. Nat. Phys. 11, 654-658 (2015).

46. Tao, Z. et al. Direct time-domain observation of attosecond final-state lifetimes in photoemission from solids. Science 353, 62-67 (2016).

47. Fidler, A. F., Singh, V. P., Long, P. D., Dahlberg, P. D. \& Engel, G. S. Dynamic localization of electronic excitation in photosynthetic complexes revealed with chiral two-dimensional spectroscopy. Nat. Commun. 5, 3286 (2017).

48. Chen, C. et al. Distinguishing attosecond electron-electron scattering and screening in transition metals. Proc. Natl Acad. Sci. USA 114, E5300-E5307 (2017)

49. Beaulieu, S. et al. Probing ultrafast dynamics of chiral molecules using time-resolved photoelectron circular dichroism. Faraday Discuss. 194, 325-348 (2016)

\section{Acknowledgements}

The experimental work was carried out at National Tsing Hua University, Institute of Photonics Technologies, supported by the Ministry of Science and Technology, Taiwan (grants 105-2112-M-007-030-MY3, 105-2112-M-001-030 and 104-2112-M-007012-MY3). The concept of isolated circularly polarized attosecond pulses was developed by C.H.-G., D.D.H., M.M.M., C.G.D., H.C.K., A.B. and A.J.-B.. C.H.-G. acknowledges support from the Marie Curie International Outgoing Fellowship within the EU Seventh Framework Programme for Research and Technological Development (2007-2013), under Research Executive Agency grant agreement no. 328334. C.H.-G. and L.P. acknowledge support from Junta de Castilla y León (SA046U16) and the Ministerio de Economía y Competitividad (FIS2013-44174-P, FIS2016-75652-P). C.H.-G. acknowledges support from a 2017 Leonardo Grant for Researchers and Cultural Creators (BBVA Foundation). M.M.M. and H.C.K. acknowledge support from the Department of Energy Basic Energy Sciences (award no. DE-FG02-99ER14982) for the concepts and experimental set-up. For part of the theory, A.B., A.J.-B., C.G.D., M.M.M. and H.C.K. acknowledge support from a Multidisciplinary University Research Initiatives grant from the Air Force Office of Scientific Research (award no. FA9550-16-1-0121). A.J.-B. also acknowledges support from the US National Science Foundation (grant no. PHY-1734006). This work utilized the Janus supercomputer, which is supported by the US National Science Foundation (grant no. CNS-0821794) and the University of Colorado, Boulder. This research made use of the high-performance computing resources of the Castilla y León Supercomputing Center (SCAYLE, www.scayle.es), financed by the European Regional Development Fund (ERDF). J.L.E. acknowledges support from the National Science Foundation Graduate Research Fellowship (DGE1144083). L.R. acknowledges support from the Ministerio de Educación, Cultura y Deporte (FPU16/02591)

\section{Author contributions}

P.-C.H., J.-T.H., P.-Y.H., C.-H.L., D.D.H., J.L.E., C.G.D., H.C.K., M.M.M., A.H.K. and M.-C.C. designed the experiment with circularly polarized isolated high-harmonic pulses. P.-C.H., J.-T.H., P.-Y.H., S.-D.Y., A.H.K. and M.-C.C. proposed the full polarization control of HHG and designed the EUV polarimeter. P.-C.H., J.-T.H., P.-Y.H., C.-H.L. and M.-C.C. performed the experiments. C.H.-G., A.B. and A.J.-B. performed the theoretical simulations on circularly polarized isolated attosecond pulses. C.H.-G., L.R. and L.P. worked on the theoretical methods and simulations of the full polarization control of HHG. P.-C.H., C.H.-G., J.-T.H., P.-Y.H., L.R., L.P. and M.-C.C. analysed data. P.-C.H., C.H.-G., L.P., M.M.M. and M.-C.C. wrote the manuscript, to which all authors suggested improvement.

\section{Competing interests}

The authors declare no competing interests.

\section{Additional information}

Supplementary information is available for this paper at https://doi.org/10.1038/ s41566-018-0145-0.

Reprints and permissions information is available at www.nature.com/reprints.

Correspondence and requests for materials should be addressed to P.-C.H. or M.-C.C.

Publisher's note: Springer Nature remains neutral with regard to jurisdictional claims in published maps and institutional affiliations. 